\title{
In vivo Polycomb kinetics and mitotic chromatin binding distinguish stem cells from differentiated cells
}

\author{
João Pedro Fonseca, ${ }^{1}$ Philipp A. Steffen, ${ }_{1}^{1}$ Stefan Müller, ${ }^{2,5}$ James Lu, ${ }^{3,5}$ Anna Sawicka, ${ }^{4}$ \\ Christian Seiser, ${ }^{4}$ and Leonie Ringrose ${ }^{1,6}$ \\ ${ }^{1}$ Institute of Molecular Biotechnology (IMBA), 1030 Vienna, Austria; ${ }^{2}$ Johann Radon Institute for Computational and Applied \\ Mathematics (RICAM), 1030 Vienna, Austria; ${ }^{3}$ Biomolecular Signaling and Control Group, Automatic Control Laboratory, Swiss \\ Federal Institute of Technology (ETH Zurich), 9082 Zurich, Switzerland; ${ }^{4}$ Max F. Perutz Laboratories (MFPL), Medical University \\ of Vienna, 1030 Vienna, Austria
}

\begin{abstract}
Epigenetic memory mediated by Polycomb group (PcG) proteins must be maintained during cell division, but must also be flexible to allow cell fate transitions. Here we quantify dynamic chromatin-binding properties of PH::GFP and PC::GFP in living Drosophila in two cell types that undergo defined differentiation and mitosis events. Quantitative fluorescence recovery after photobleaching (FRAP) analysis demonstrates that PcG binding has a higher plasticity in stem cells than in more determined cells and identifies a fraction of PcG proteins that binds mitotic chromatin with up to 300 -fold longer residence times than in interphase. Mathematical modeling examines which parameters best distinguish stem cells from differentiated cells. We identify phosphorylation of histone $\mathrm{H} 3$ at Ser 28 as a potential mechanism governing the extent and rate of mitotic PC dissociation in different lineages. We propose that regulation of the kinetic properties of PcG-chromatin binding is an essential factor in the choice between stability and flexibility in the establishment of cell identities.
\end{abstract}

[Keywords: Polycomb; Polyhomeotic; Drosophila; mitosis; kinetics; stem cell]

Supplemental material is available for this article.

Received December 22, 2011; revised version accepted March 6, 2012.

Polycomb group (PcG) proteins play a crucial role in maintaining the repressed transcriptional states of their target genes in stem cells and differentiated cells (Sawarkar and Paro 2010). Cell division is a vital moment for the action of PcG proteins. The vast majority of chromatinbinding proteins dissociate from mitotic chromatin, and there is a global transcriptional shutdown (Prescott and Bender 1962; Martinez-Balbas et al. 1995; Gottesfeld and Forbes 1997). However, for the PcG target genes, it is through mitosis that the memory of transcriptional status must be maintained, and it is also during mitosis that an opportunity for flexibility in PcG target status exists, enabling changes of cell identity during differentiation. Thus, to better understand memory and flexibility,

\footnotetext{
${ }^{5}$ These authors contributed equally to this work.

${ }^{6}$ Corresponding author.

E-mail leonie.ringrose@imba.oeaw.ac.at.

Article is online at http://www.genesdev.org/cgi/doi/10.1101/gad.184648.111.

Freely available online through the Genes \& Development Open Access option.
}

it is essential to study the behavior of PcG proteins during mitosis and differentiation.

Genome-wide profiling indicates that fly and vertebrate PcG proteins change their targets dynamically upon differentiation (Mohn et al. 2008; Schuettengruber and Cavalli 2009). Consistent with this, live imaging studies have documented changes in subcellular localization and PcG protein mobility upon differentiation /Chen et al. 2005; Ren et al. 2008) or at different stages of development (Ficz et al. 2005). However, a detailed quantitative analysis of the behavior of PcG proteins in living animals in a single-cell system with defined differentiation processes and mitotic activity is lacking. Binding of fly and vertebrate PcG proteins to mitotic chromatin has been observed in some cell types (Fanti et al. 2008; Vincenz and Kerppola 2008), while other studies report substantial dissociation (Buchenau et al. 1998; Dietzel et al. 1999). These differences may arise from the use of different techniques or may be species-specific or cell type-specific. Thus, whether there are quantitative changes in the nature 
of PcG binding to mitotic chromatin in different cell types of a single animal is not clear.

Here we investigate the behavior of GFP-fused Polycomb (PC::GFP) and Polyhomeotic (PH::GFP) in living Drosophila larval neuroblasts and pupal sensory organ precursor cells (SOPs). Neuroblasts are stem cell neuronal progenitors that divide asymmetrically to give origin to another neuroblast and a ganglion mother cell (GMC) (Doe 2008). SOPs are more determined cells that arise later in fly development and divide asymmetrically to give two well-defined daughter cells, pIIa and pIIb (Neumuller and Knoblich 2009). We use a combination of quantitative live imaging and mathematical modeling to query the interaction of PcG proteins with chromatin in the neuroblast and SOP lineages both in interphase and on metaphase chromosomes. We show that PcG protein mobility is decreased in more differentiated cells. In addition, we identify and quantify a fraction of mitotically bound PcG proteins whose chromatin-binding properties are profoundly different from those measured in interphase, showing up to 300-fold longer residence times. We identify mitotic phosphorylation of histone $\mathrm{H} 3$ at Ser 28 as a potential mechanism governing the extent and rate of mitotic PC dissociation. These findings suggest that mitosis provides a unique time frame in the cell cycle where the PcG system chooses between stability and flexibility during the establishment of cell identities.

\section{Results}

Transgenic PC::GFP and PH::GFP fulfill the functions of the endogenous proteins

To investigate the behavior of the Polycomb-repressive complex 1 (PRC1) proteins $\mathrm{PC}$ and $\mathrm{PH}$ during cell division and differentiation, we studied previously characterized EGFP fusions of these proteins (Dietzel et al. 1999; Ficz et al. 2005). (EGFP is henceforth referred to as GFP.) The PH::GFP fusion protein rescues homozygous ph-null alleles (Ficz 2005; Ficz et al. 2005) and thus can replace the endogenous protein. The PC::GFP fusion protein has been reported to rescue homozygous alleles carrying mutations in the PC chromodomain, such as $P c^{X L 5}$, but not homozygous null alleles, such as $P c^{3}$, or those that produce C-terminally truncated protein (Dietzel et al. 1999|. Thus the PC::GFP fusion can partially fulfill the functions of the endogenous protein in a $P c$ homozygous mutant context. The suitability of this fusion protein for live imaging studies had been addressed by several investigators, demonstrating that the PC::GFP fusion protein binds chromatin and participates in the PRC1 complex. This is supported by the banding pattern of PC::GFP on polytene chromosomes in both intact salivary gland nuclei (Dietzel et al. 1999; Ficz et al. 2005) and fixed preparations (Ficz et al. 2005). In addition, the genome-wide distribution of PC::GFP shows good agreement with that of endogenous PC (Kwong et al. 2008). Further supporting evidence for the correct chromatinbinding behavior of PC::GFP is the timing of mitotic dissociation and reassociation (Dietzel et al. 1999), which is identical to the distribution of PC measured for the endogenous PC protein determined by immunofluorescence (Buchenau et al. 1998).

Nevertheless, to further characterize the PC::GFP fusion protein, we re-examined its ability to rescue transheterozygous $P c$ mutants. Previous rescue experiments have been performed in homozygous mutant backgrounds (Dietzel et al. 1999). Since chromosomes carrying lethal mutations are maintained as heterozygote stocks over balancer chromosomes, they may accumulate second site mutations that lead to lethality when the chromosome is brought into the homozygous state in the rescue experiment. We did not observe rescue of transheterozygous combinations of null alleles; however, this analysis showed that the transheterozygous lethal combination of $P c^{3} / P c^{X L 5}$ was rescued to adulthood by the PC::GFP transgene (Fig. 1A). Thus, the PC::GFP transgene can rescue lethality in a more severely compromised genetic background than previously shown.

In the following experiments, we examine the behavior of PH::GFP and PC::GFP in neuroblasts of third instar larval brains and in SOPs of pupae. PcG expression is essential for neuroblast survival (Bello et al. 2007; Neumuller et al. 2011) and for normal development of SOPs and sensory organs (Sharp et al. 1994; MummeryWidmer et al. 2009). We therefore asked whether the expression of an additional transgenic copy of $\mathrm{PC}$ or $\mathrm{PH}$ would adversely affect development in these lineages. PC::GFP was expressed under the control of the ubiquitous PC promoter (Dietzel et al. 1999), whereas PH::GFP was expressed from a UAS transgene (Ficz et al. 2005). In addition to the UAS sequence, the ph::gfp construct contains the Pc promoter; however, this promoter gives expression of PH::GFP only in salivary glands (Ficz et al. 2005). To drive expression of PH::GFP specifically in the neuroblast and SOP lineages, we used the worniu and neuralized GAL4 drivers. Worniu is active in all neuroblasts of the larval brain but not in neuroblast progeny (Lee et al. 2006), in addition to expression in the embryonic CNS (Wheeler et al. 2006). neuralized is expressed in the SOP and its progeny cells (Bellaiche et al. 2001). It has been demonstrated that overexpression of $\mathrm{PH}$ leads to cell death and tissue abnormalities (Ficz 2005; Martinez et al. 2009). The UAS-GAL4 expression strategy for $\mathrm{PH}:$ :GFP was intended to circumvent this problem by expressing the transgene during a limited developmental window.

In order to test whether even this limited expression strategy would nevertheless disrupt the lineages of interest, we examined the morphology and number of neuroblasts in larval brains and the morphology of sensory organs in adult flies. This analysis was performed for both PH::GFP and PC::GFP. Despite readily visible GFP signals, transgenic animals expressing either PH::GFP or PC::GFP showed normal larval brain morphology (Fig. 1B-D) and no detectable change in the number or morphology of neuroblasts (Fig. 1E-G). Likewise, in adults, no change in the number or morphology of sensory organs was detected in transgenic animals (Fig. 1H-J). We therefore conclude that the additional transgenic copy of $\mathrm{PH}$ or 
PC does not adversely affect the development of the neuroblast and SOP lineages.

To determine expression levels of transgenes, we used quantitative Western blotting. Quantification of transgenic PH::GFP by Western blot was not possible due to the driver-inducible neuroblast- and SOP-specific expres-
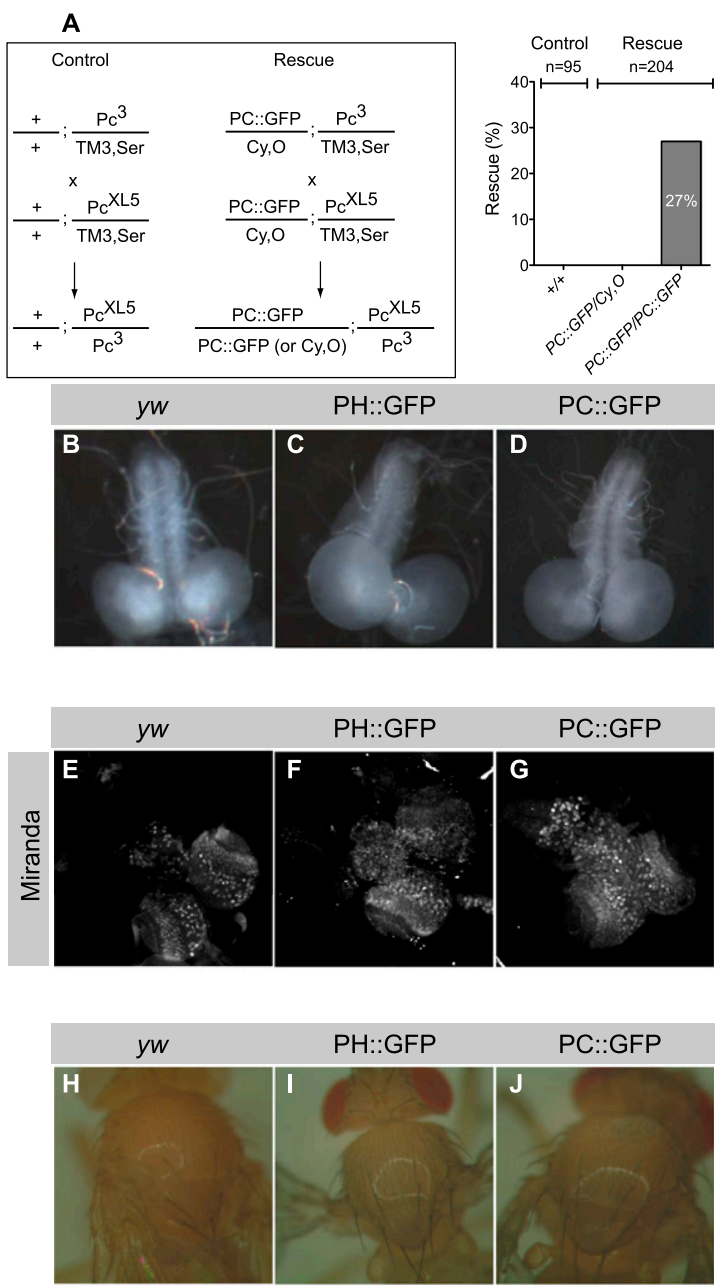

$\mathbf{K}$

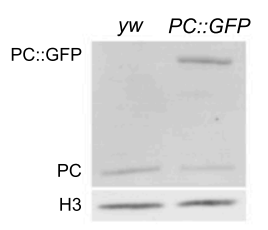

M

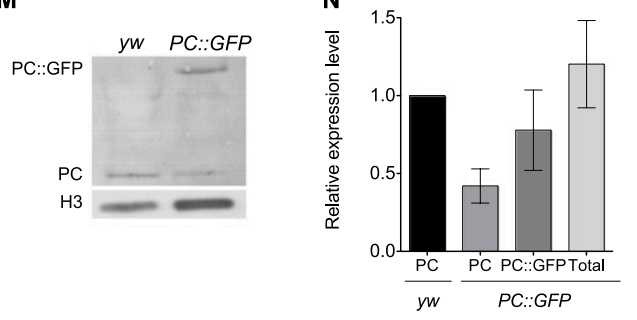

sion of PH::GFP, which would require isolation of cell type-specific protein extracts. However, since PC::GFP is expressed under the control of the ubiquitous PC promoter, we reasoned that whole-tissue extracts of larval brains (Fig. 1K,L) and whole pupae (Fig. 1M,N) would be informative. Interestingly, the endogenous PC protein was substantially down-regulated in the presence of the transgene in both tissues, leading to a total PC overexpression of twofold in brains (Fig. 1L) and 1.2-fold in pupae (Fig. 1N). Similar down-regulation of the endogenous proteins in the presence of $\mathrm{PH}: \mathrm{GFP}$ or PC::GFP transgenes in wing discs and embryos has been reported previously (Ficz et al. 2005). Thus, despite the presence of the additional transgenic copy, the total protein levels are near to endogenous levels. In addition, since they rescue mutants and do not adversely affect development, we conclude that both the PH::GFP and PC::GFP transgenes are useful reporters of endogenous protein behavior.

\section{Quantitative live imaging of PcG proteins in single defined cell lineages reveals transitions in protein concentration upon entry into mitosis}

To examine the behavior of PC::GFP and PH::GFP during mitotic division in neuroblasts and SOPs, we performed live-cell imaging on neuroblasts in whole larval brains (Fig. 2; Supplemental Movies S1, S2) and the SOP lineage in pupal notum (Fig. 3; Supplemental Movies S3, S4). To quantify GFP signal intensities, a three-dimensional (3D)

Figure 1. Analysis of $\mathrm{PC}$ and $\mathrm{PH}$ transgenes. $(A)$ Rescue of $P C^{X L 5} / P c^{3}$ mutants by PC::GFP expression. Progeny of the crosses between $+; P c^{X L 5} / T M 3, S b$ and $+; P c^{3} / T M 3, S b$ and between $P c:: G F P / C y O ; P c^{X L 5} / T M 3, S b$ and $P c:: G F P / C y O ; P^{3} /$ $T M 3, S b$ were scored for the number of adult flies for which the third chromosome was $P c^{X L 5} / P c^{3}$, and the proportion of these for which the second chromosome was heterozygous for the transgene $(P C:: G F P /+)$ or homozygous for the transgene $(P C:: G F P / P C:: G F P)$ was calculated. The expected number of flies of each genotype in the event of a full rescue was calculated. (\% rescue) The percentage of this expected number that was represented by flies of that genotype. Above the plot are shown the total number of flies counted in each experiment. $(B-I)$ Transgenic PH::GFP and PC::GFP do not adversely affect the neuroblast and SOP lineages. $(B-D)$ Third instar larval brains showing normal morphology in the presence of either PH::GFP $(C)$ or PC::GFP $(D)$ in comparison with control yw brains $(B)$. (EG) Third instar larval brains were stained with $\alpha$ Miranda antibody (see the Materials and Methods) to visualize neuroblasts, showing no detectable change in neuroblast number and localization in the presence of either transgene. $(H-J)$ Adult nota of $y w(H), P H:: G F P(I)$, and $P C:: G F P(J)$ flies are shown, showing no detectable change in sensory organ number and morphology in transgenic flies. $(K, M)$ Western blot using $\alpha \mathrm{PC}$ antibody to detect endogenous and GFP-fused PC in extracts of third instar larval brains $(K)$ and whole pupae $(M)$ of $y w$ and $P C:: G F P$ flies. Histone $\mathrm{H} 3$ was used as loading control. $(L, N)$ Quantification of Western blots in $K$ and $M$, performed as described in the Materials and Methods. Data show the mean and standard error of three independent Western blots. 
Fonseca et al.
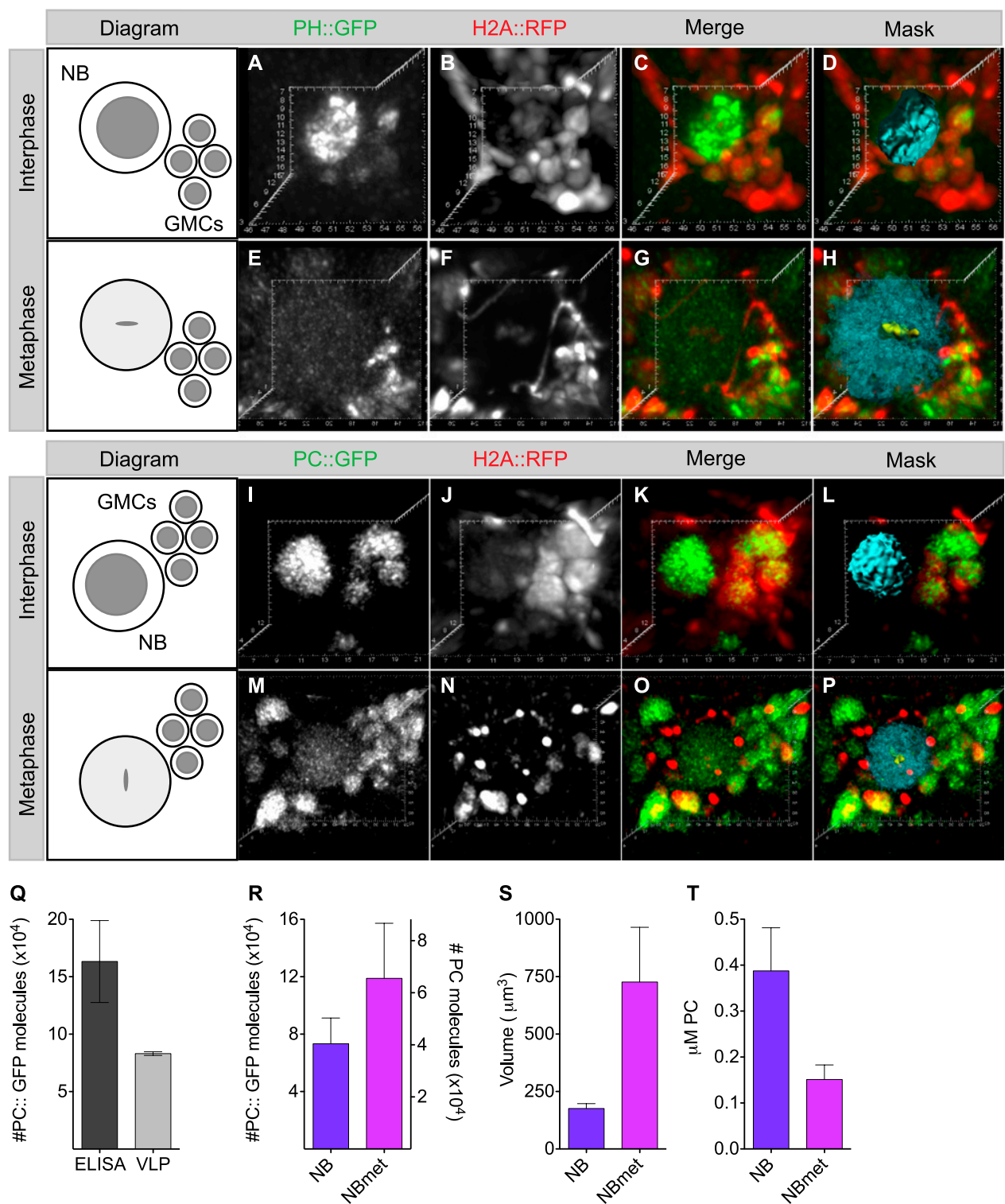

Figure 2. Quantification of PH::GFP and PC::GFP in neuroblast lineage. $(A-P)$ Deconvolved confocal laser scanning images of PH::GFP $(A, E)$, PC::GFP $(I, M)$, and H2A::RFP $(B, F, J, N)$ in neuroblast interphase $(A-D, I-L)$ and metaphase $(E-H, M-P)$. (Left) Diagrams identify neuroblast (NB) and GMCs in images. $(C, G, K, O)$ Merge panels show PH::GFP $(C, G)$ or PC::GFP $(K, O)$ in green and H2A::RFP in red. $(D, H, L, P)$ Mask panels show the volumes occupied by PH::GFP $(D, H)$ and PC::GFP $(L, P)$ in blue and the volume occupied by H2A::RFP in yellow. Neuroblast lineage-specific expression of PH::GFP and H2A::RFP was obtained by using a worniu-GAL4 driver. For PC::GFP imaging, PC::GFP was expressed under the endogenous $P c$ promoter, and H2A RFP was expressed from the ubiquitin promoter (see the Materials and Methods for genotypes). (Q) Estimated number per nucleus of PC::GFP molecules in blastoderm embryos (2-3 h) using GFP detection by ELISA (Cell Biolabs) or by GFP-VLP calibration, as described in the Materials and Methods. Data show the mean and $95 \%$ confidence intervals of 10 cells for GFP-VLP measurements and 10 independent ELISA assays. $(R)$ Estimated number per cell of PC::GFP molecules (left Y-axis) and endogenous PC molecules (right Y-axis) in yw flies by GFP-VLP calibration in neuroblast interphase (NB) and metaphase (NBmet). Data show the mean and 95\% confidence interval of at least four independent measurements. (S) Measured cell volume of neuroblasts in interphase (NB) and metaphase (NBmet). Data show the mean and $95 \%$ confidence intervals of at least four cells. (T) Estimated micromolar concentrations of endogenous PC in yw flies in neuroblast interphase (NB) and metaphase (NBmet).

mask of the volume occupied by the GFP fusion protein in the cell of interest was applied, and signal intensities were calculated (Figs. 2D,H,L,P, 3D,H,L,P,T,X). In order to convert signal intensities to absolute concentrations of GFP fusion proteins in single cells, we performed calibra- tion using GFP-containing virus-like particles (GFPVLPs) (Figs. 2Q,R, 3Y; Supplemental Table S1; Dundr et al. 2002; Rabut et al. 2004). To evaluate the accuracy of GFP-VLP calibration for live GFP quantification, we compared this technique with GFP ELISA (enzyme-linked 


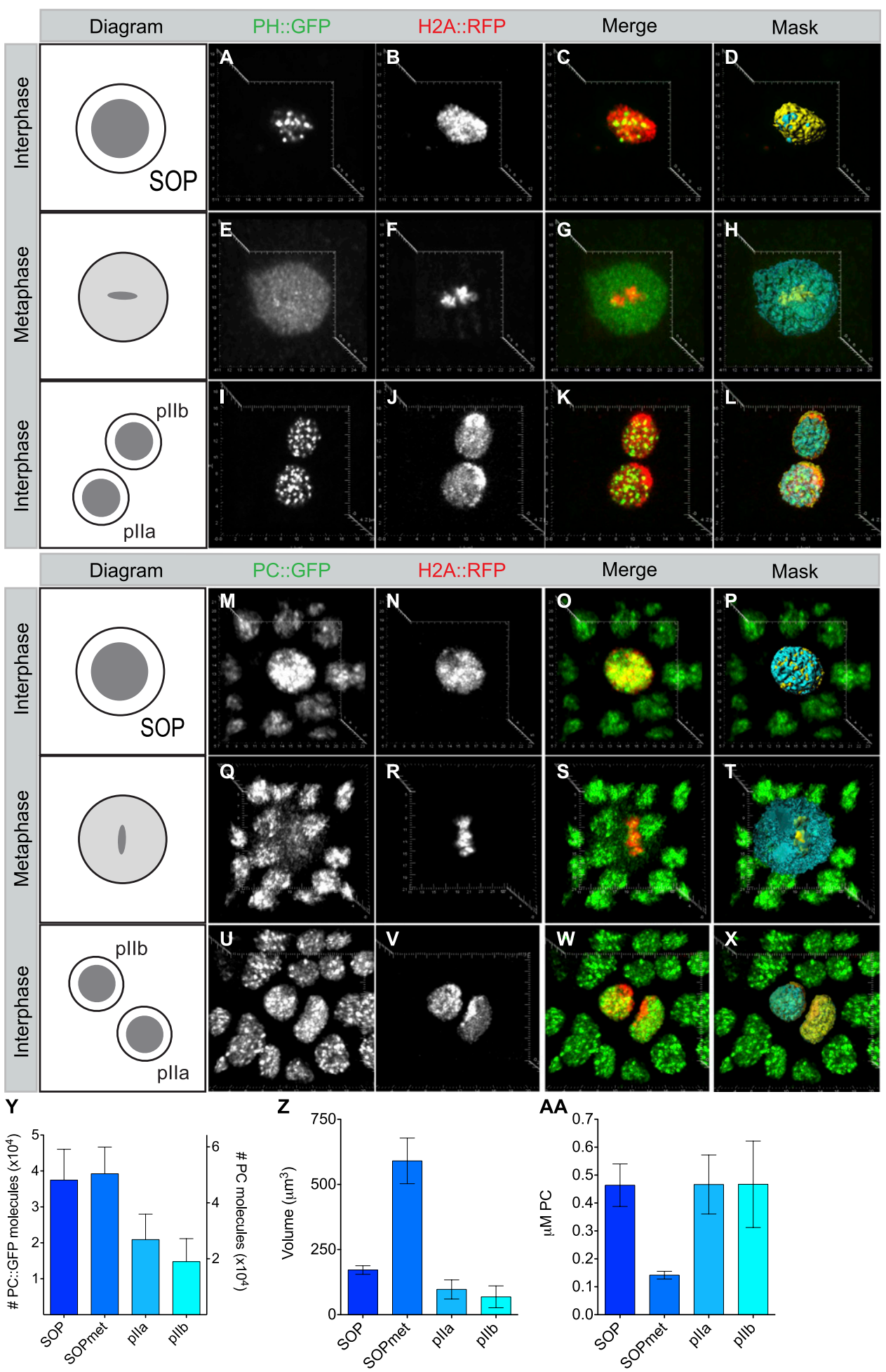

Figure 3. Quantification of $\mathrm{PH}:: \mathrm{GFP}$ and PC::GFP in SOP lineage. $(A-X)$ Deconvolved confocal laser scanning images of PH::GFP $(A, E, I)$, PC::GFP $(M, Q, U)$, and H2A::RFP $(B, F, J, N, R, V)$ in SOP interphase $(A-D, M-P)$ and metaphase $(E-H, Q-T)$ and pIIa and pIIb interphase $(I-L, U-X)$. (Left) Diagrams identify SOPs and their daughter cells in images. $(C, G, K, O, S, W)$ Merge panels show PH::GFP $(C, G, K)$ or PC::GFP $(O, S, W)$ in green and H2A::RFP in red. $(D, H, L, P, T, X)$ Mask panels show the volumes occupied by PH::GFP $(D, H, L)$ and PC::GFP $(P, T, X)$ in blue and the volume occupied by H2A::RFP in yellow. SOP lineage-specific expression of PH::GFP and H2A::RFP was obtained by using a neuralized-GAL4 driver. For PC::GFP imaging, PC::GFP was expressed under the endogenous PC promoter.|see the Materials and Methods for genotypes). (Y) Estimated number per nucleus or metaphase cell of PC::GFP molecules (left Y-axis) and endogenous PC molecules (right Y-axis) in yw flies measured by GFP-VLP calibration in SOP interphase (SOP) and metaphase (SOPmet) and pIIa and pIIb interphase. Data show the mean and $95 \%$ confidence intervals of at least three cells. $(Z)$ Measured cell volumes of different cells in SOP lineage. Data show the mean and 95\% confidence intervals of at least three cells. $(A A)$ Estimated micromolar concentrations of endogenous PC in yw flies, per nucleus or metaphase cell in different cells of SOP lineage. 
immunosorbent assay) by applying both quantification methods to blastoderm embryos (Fig. 2Q; see the Materials and Methods; Supplemental Material). This analysis showed an approximately twofold difference between the ELISA and VLP measurements, probably due to diffraction of the GFP signal caused by the thickness of the embryo. We conclude that live GFP quantification by GFP-VLP calibration gives a meaningful readout of protein concentrations for single-cell experiments.

By performing live GFP quantification and GFP-VLP calibration for PH::GFP and PC::GFP in each cell lineage at interphase and metaphase (Figs. 2, 3), we calculated the number of GFP fusion protein molecules in each cell type (Figs. 2, 3; Supplemental Table S1). Conversion to micromolar concentrations via the measured volume occupied by GFP in each cell type revealed that both GFP fusion proteins undergo a substantial dilution (between 2.6-fold and 5.2-fold) (see Supplemental Table S1) upon the interphase-to-metaphase transition in both cell lineages due to the increase in volume upon nuclear envelope breakdown (NEBD) (Figs. 2S, 3Z; Supplemental Table S1). Since the PC::GFP expression strategy allowed estimation of endogenous PC protein levels (Fig. 1), we were able to use measured PC::GFP molecule numbers and concentrations to calculate the expected values for the endogenous PC protein in the wild-type situation (Figs. 2R,T, $3 Y, A A)$. This analysis revealed different absolute numbers of endogenous PC molecules calculated in interphase for the neuroblast (Fig. 2R), the SOP, and the SOP daughter cells pIIa and pIIb (Fig. 3Y). Remarkably, however, due to the different nuclear volumes of these four different cell types (Figs. 2S, 3Z), the calculated micromolar concentration of endogenous PC was essentially identical in all four cell types, at $\sim 0.4 \mu \mathrm{M}$ (Figs. 2T, 3AA). In summary, this quantitative analysis of single defined cell lineages reveals substantial dilution of $\mathrm{PC}$ and $\mathrm{PH}$ upon entry into mitosis, but a remarkably similar endogenous concentration of PC at interphase in both lineages.

\section{PC shows more dynamic interaction with chromatin than $\mathrm{PH}$}

Previous studies have determined similar dissociation rates for PH::GFP and PC::GFP from interphase chromatin in wing discs (Ficz 2005; Ficz et al. 2005). However, studies of mitotic dissociation showed distinct behavior for the two proteins upon reassociation to chromatin after mitosis (Buchenau et al. 1998). We thus asked whether PC and $\mathrm{PH}$ interact differently with chromatin in neuroblasts and SOPs and whether different cell cycle stages are distinguished by different chromatin-binding properties of either protein. To this end, we performed fluorescence recovery after photobleaching (FRAP) on both GFP fusion proteins in each cell type at interphase and metaphase (Fig. 4). Histone H2A fused to RFP (H2A::RFP) was used to mark chromatin in both lineages (Figs. 2, 3) and served as a guide for bleach spot placement on metaphase chromatin.

The recovery data were analyzed by fitting kinetic models (McNally 2008; Mueller et al. 2008, 2010). Comparison of different fitting procedures showed contributions of both diffusion and binding to the recovery kinetics (Supplemental Fig. S1). Thus the reaction-diffusion model of Mueller et al. (2008) was used for all data sets, enabling values to be extracted for the diffusion coefficient $(D f)$, the pseudo-first-order association rate $\left(k^{*}{ }_{\text {on }}\right)$, and the dissociation rate $\left(k_{\text {off }}\right)$ (Fig. 4). To evaluate the robustness of the extracted values, these parameters were cross-validated by several independent means (see Supplemental Material [FRAP Data Analysis]). This cross-validation analysis confirmed that a homogeneous global binding model is sufficient to describe both the interphase and metaphase data (Supplemental Fig. S2; Beaudouin et al. 2006; Sprague et al. 2006), provided independent measures for $D f$ that are in excellent agreement with those extracted from fitting (Supplemental Fig. S3), and defined limits for the extracted values of $k^{*}$ on and $k_{\text {off }}$ for each data set (Supplemental Fig. S4).

Comparison of the recovery kinetics and measured parameters for PC::GFP and PH::GFP revealed striking differences between the two proteins. PH::GFP typically showed far slower recovery than PC::GFP (Fig. 4A-F). This was due in part to a slower $D f$ determined for PH::GFP than for PC::GFP (Supplemental Fig. S3; Supplemental Table S1). Further differences between PC::GFP and PH::GFP were observed upon comparison of the bound fractions and kinetic rate constants. Calculation of the percentage of total protein bound at interphase in different cell types showed $30 \%-70 \%$ of total PH::GFP bound, in contrast to $10 \%-20 \%$ of total PC::GFP bound (Fig. 4G; Supplemental Table S1). We note that one caveat of these comparisons is that the percentage of total $\mathrm{PH}$ protein represented by $\mathrm{PH}:$ :GFP is unknown. Nevertheless, the comparison of residence times-which reflect an inherent, concentration-independent property of proteinchromatin binding-revealed twofold to ninefold longer residence times for $\mathrm{PH}:$ GFP than PC::GFP at interphase in all cell types (Fig. 4I; Supplemental Table S1). Taken together, these results demonstrate inherently different behaviors of PC::GFP and PH::GFP in terms of both diffusion and chromatin interaction, with PC::GFP showing faster diffusion and more dynamic interaction with chromatin than PH::GFP.

\section{PcG proteins show decreased mobility upon lineage commitment}

Previous studies of the kinetics of PH::GFP chromatin binding in Drosophila at different developmental stages have documented an approximately twofold lower average dissociation rate in larval wing imaginal discs than in embryos, indicating longer residence times and thus suggesting more stable chromatin association as development proceeds (Ficz et al. 2005).

To evaluate whether PcG proteins interact differently with chromatin in different cell lineages and in the progressive commitment of a single lineage, we compared kinetic parameters at interphase in neuroblasts and the SOP lineage (Fig. 4G-I). For PH::GFP, we measured an approximately twofold lower dissociation rate constant, giving a twofold longer residence time in SOP interphase 

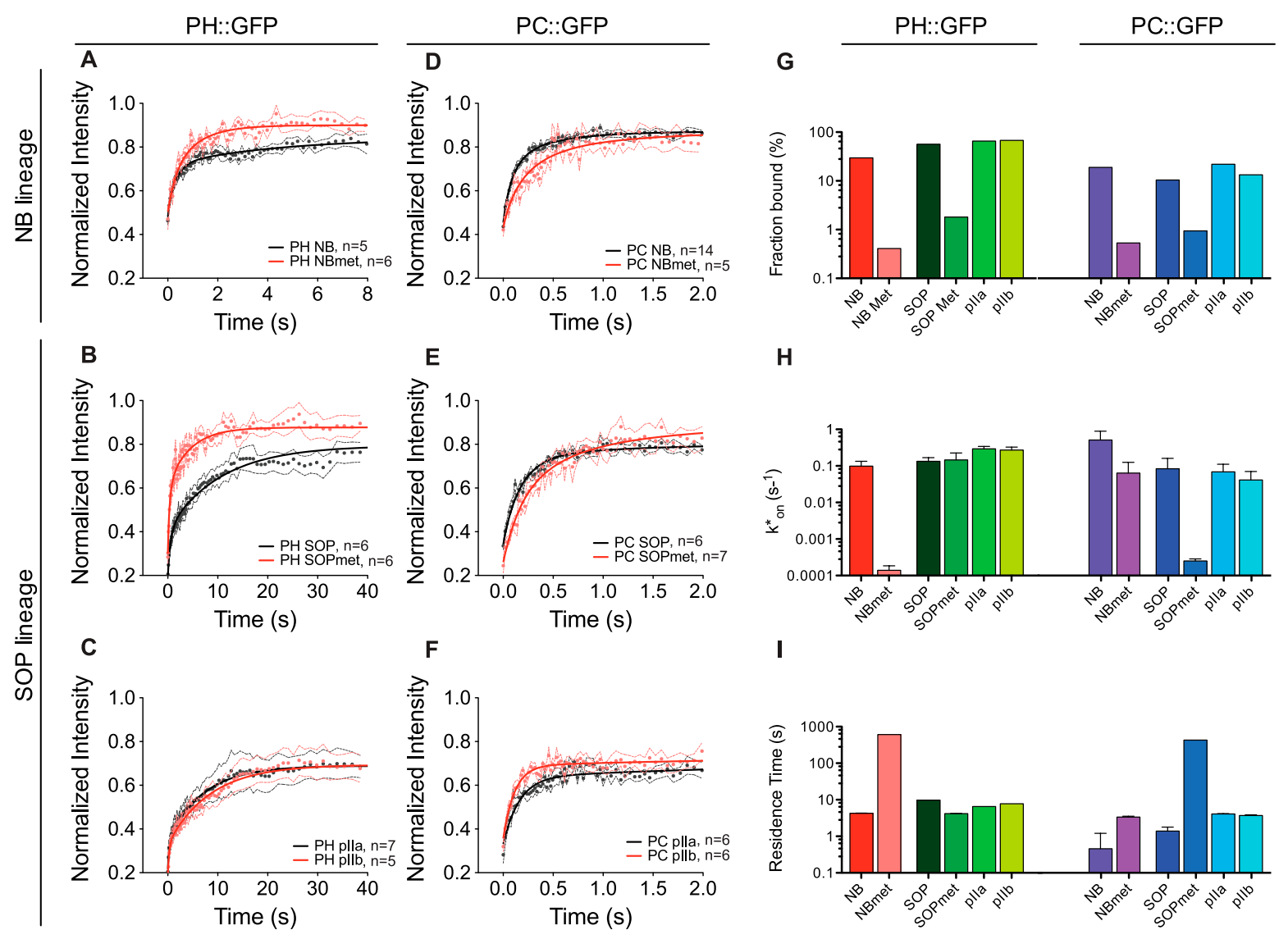

H
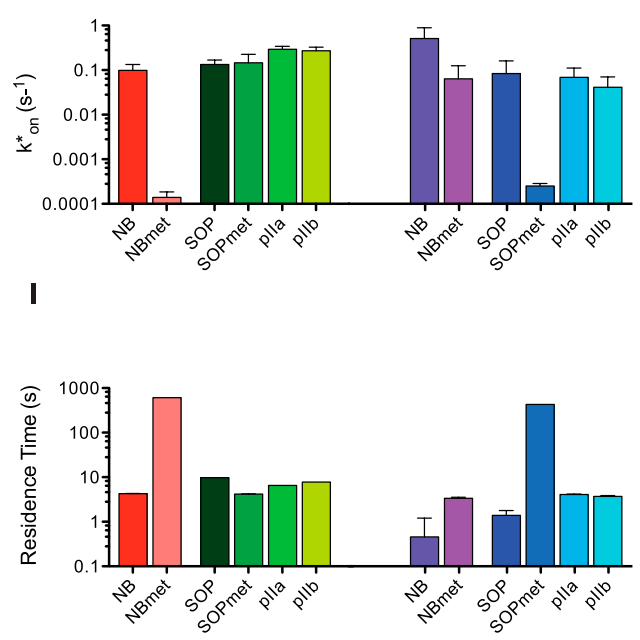

Figure 4. FRAP analysis of $\mathrm{PH}:: \mathrm{GFP}$ and $\mathrm{PC}:: \mathrm{GFP}$ in neuroblast $(\mathrm{NB})$ and SOP lineages. $(A-F)$ FRAP recovery curves of $\mathrm{PH}:: \mathrm{GFP}(A-C)$ and PC::GFP $(D-F)$ in the neuroblast $(\mathrm{NB})(A, D)$ and SOP $(B, C, E, F)$ lineages. Interphase bleach spots were placed to cover several PC::GFP or PH::GFP foci (see Figs. 2, 3). Metaphase bleach spots were placed in the region of the RFP signal (see Figs. 2, 3). Symbols represent the mean of experimental values and are accompanied by the standard error of the mean, represented by a dashed line of the same color. The solid darker line shows fit of FRAP model to the experimental data (see the Materials Methods; Supplementary Material [FRAP Data Analysis], for full description of model and fitting procedures). NB and SOP (black on $A, B, D, E)$ indicate neuroblast and SOP interphase, and NBmet and SOPmet (red on $A, B, D, E)$ indicate neuroblast and SOP metaphase. pIIa (black in $C, F)$ and pIIb $($ red in $C, F)$ indicate the interphase of the respective SOP daughter cells. $(G-I)$ Extracted total fraction bound $(G)$, pseudo-first-order association rate $k^{*}{ }_{\text {on }}(H)$, and residence time $(I)$ of PH::GFP and PC::GFP in neuroblast and SOP lineages from FRAP model fit. The fraction bound in the chromatin region was calculated as $k^{*}{ }_{\text {on }} /\left(k_{\text {off }}+k^{*}{ }_{\text {on }}\right)$. For metaphase measurements, the fraction bound in the chromatin region was used in combination with the quantity of free protein outside the chromatin volume to calculate the fraction of total protein bound, shown on the plots (see also Supplemental Table S1). Residence time was calculated as $1 / k_{\text {off. }}$. Error bars show $95 \%$ confidence intervals.

compared with neuroblast interphase (Fig. 4I; Supplemental Table S1). In the more determined daughters of the SOP (pIIa and pIIb), no further increase in residence time was detected. However, the association rate constant was approximately twofold higher in these daughter cells, contributing to a substantial increase in the calculated bound fraction from $57 \%$ in SOPs to $66 \%-$ $68 \%$ in the daughters (Fig. 4G; Supplemental Table S1). For PC::GFP, a similar trend was observed. We measured a threefold longer residence time at interphase in SOPs than in neuroblasts and a further threefold increase in residence time in pIIa and pIIb (Fig. 4I; Supplemental Table S1). Taken together, these results indicate not only that the interaction of both PC and PH with chromatin is more dynamic in the stem cell neuroblast than in the SOPs, but also that the mobility of both proteins de- creases upon increasing cell fate commitment within the SOP lineage.

\section{A fraction of PcG proteins remains strongly bound to mitotic chromatin}

To determine whether the nature of PcG-chromatin interactions changes during the cell cycle, we examined metaphase binding. Calculation of bound fractions of PH::GFP and PC::GFP from FRAP analysis of metaphase chromatin showed a clear reduction in binding, with $0.4 \%-2 \%$ of total protein remaining bound to metaphase chromosomes in both cell lineages (Fig. 4G; Supplemental Table S1). Independent calculation of metaphase-bound fractions based on image quantification gave consistent results (Supplemental Table S1). Thus, 
a small fraction of PcG proteins remains bound to mitotic chromatin.

To assess the kinetic properties of this fraction, we examined association rates and residence times (Fig. 4H,I; Supplemental Table S1). Remarkably, in most cases, this metaphase-bound population showed properties profoundly different from that bound in interphase, in terms of both a slower association rate (Fig. $4 \mathrm{H}$ ) and a longer residence time (Fig. 4I). For each protein, the extent of kinetic changes was different in different lineages. For example, PH::GFP showed no significant change in behavior between interphase and metaphase in SOPs, but a profound change in neuroblasts (Fig. 4H,I; Supplemental Table S1). For PC::GFP, the residence time in neuroblasts increased 7.3 -fold, from $0.46 \mathrm{sec}$ in interphase to $3.35 \mathrm{sec}$ in metaphase. In contrast, in SOPs for the same protein, the increase in residence time at metaphase was $>300$ fold, from $1.39 \mathrm{sec}$ in interphase to $>5 \mathrm{~min}$ in metaphase (Supplemental Table S1). To determine the robustness of the extracted parameters, we performed simulations of FRAP recovery for a range of $k^{*}$ on and $k_{\text {off }}$ values and evaluated the fit to the experimental data (Supplemental Fig. S4). In both lineages and for both proteins, the $k^{*}$ on and $k_{\text {off }}$ combinations giving the best fits to the metaphase and interphase data occupied distinct parameter spaces (Supplemental Fig. S4), indicating robust differences between the measured data sets between interphase and metaphase. These protein- and lineage-specific differences in kinetic behavior suggest that the changes in

A

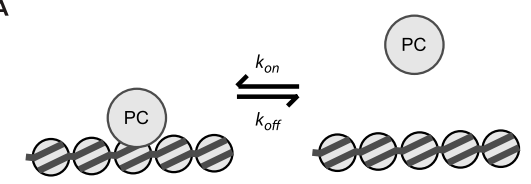

B

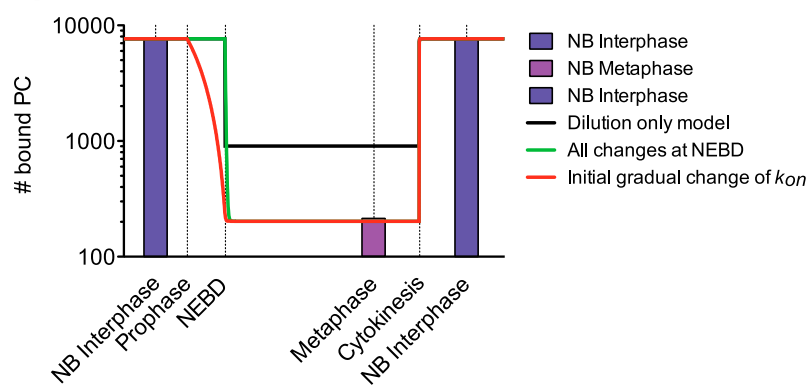

C

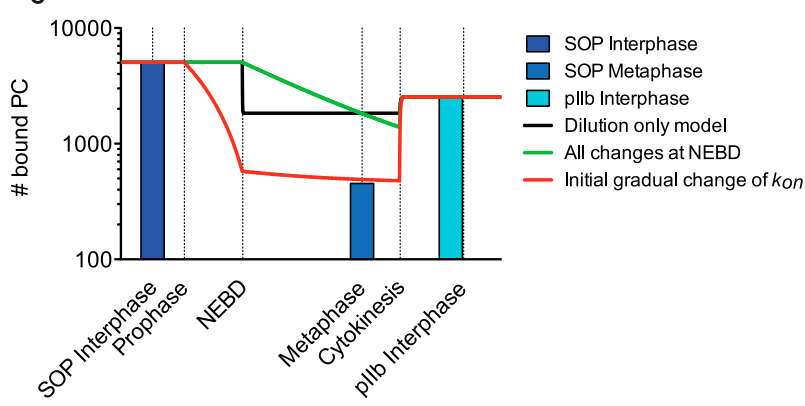

binding properties at metaphase are not a general effect of chromatin condensation at mitosis but are specific to each protein in each cell type.

\section{Mathematical modeling predicts a slower time scale for mitotic dissociation of PC in SOPs than in neuroblasts}

In the above experiments, we measured different chromatin-binding properties of PcG proteins at specific points during the cell cycle; namely, interphase and metaphase. We next asked whether the changes in kinetic properties that we measured are sufficient to account for the observed reduction in binding at metaphase within the known time frame of cell division in SOPs and neuroblasts (Mayer et al. 2005; Lee et al. 2006). To this end, we used mathematical modeling to simulate a time course of protein-chromatin interactions upon cell cycle transitions. The PcG-chromatin interaction was modeled using ordinary differential equations (Fig. 5A; Supplemental Material [Mathematical Modeling]). As inputs, we used the calculated numbers of endogenous PC molecules in SOPs and neuroblasts, the association and dissociation rates measured in FRAP at interphase and metaphase in each cell lineage, and the corresponding nuclear or cell volumes (Supplemental Table S1).

Figure 5. Mathematical modeling of mitotic dissociation of PC from chromatin. (A) Model for interaction of PC protein with chromatin targets. PC interacts with chromatin with the association rates $k_{\text {on }}$ and dissociation rates $k_{\text {off }}$. The model describes three species: chromatin-bound PC, free PC, and free chromatin. Implementation of the model is described in the Supplemental Material, (Mathematical Modeling). (B,C) Modeling of endogenous PC in neuroblast $(B)$ and SOP $(C)$ lineages of $y w$ flies. Time is scaled in real time according to Mayer et al. (2005) and Lee et al. (2006). Solid bars represent calculated numbers of bound PC molecules extracted from FRAP experiments at interphase or metaphase in each cell type as indicated. Solid lines represent bound numbers of PC molecules predicted by simulation using the model shown in $A$ under different assumptions as follows: (Black line) Dilution only model: Dilution of PC as measured (Figs. 2T, 3AA) occurs at NEBD, but changes in binding rates are not included in the simulation to evaluate the contribution of dilution alone to the chromatinbinding properties of the system. This model predicts a substantial dissociation of PC caused by dilution alone in both cell types (shown in $B, C$ ), which nevertheless fails to reach the measured levels of dissociation. (Green) All changes at NEBD. The measured dilution (Figs. 2T, 3AA) and extracted changes in binding parameters (Fig. $4 \mathrm{H}, \mathrm{I}$ ) are included in the model, with all changes occurring simultaneously at NEBD. For neuroblasts $(A)$, this model predicts a rapid dissociation to measured levels, whereas for SOPs $(B)$, the same model using SOP parameters predicts a gradual dissociation, which does not reach measured levels of dissociation by the onset of cytokinesis. (Red) Initial gradual change of $k_{\text {on }}$. The model assumes dilution as measured (Figs. 2T, 3AA) at NEBD and change in $k_{\text {off }}$ as measured (Fig 4I) at NEBD, but the association rate changes gradually from extracted interphase to metaphase values (Fig $4 \mathrm{H}$ ) from prophase to NEBD. This model predicts dissociation of PC in SOPs that is close to measured levels (shown in $B$ ). 
We first asked whether a change in the kinetic properties of PC is actually required for protein dissociation at metaphase or whether the simple effects of protein dilution at NEBD would be sufficient (Fig. 5B,C, black lines). These simulations predicted that although dilution alone would achieve a substantial degree of dissociation, the measured levels of metaphase binding are an order of magnitude lower still. We next asked whether the changes in kinetic rate constants that we measured would be sufficient to remove this additional protein from mitotic chromosomes within the time frame imposed by the cell cycle (Fig. 5B,C, green lines). For this simulation, the dilution and changes in binding properties were all effected concomitantly upon NEBD. This simulation predicted a rapid dissociation to the observed levels within seconds of NEBD in neuroblasts. However, the predicted dissociation in SOPs was very slow, such that the observed levels were not reached before the cell entered cytokinesis at the end of metaphase (Fig. 5C). In the simulation, the system reached equilibrium after $\sim 33 \mathrm{~min}$, predicting 385 bound molecules (data not shown). Further inspection of parameters revealed that this difference between the cell types was independent of diffusion (Supplemental Material [Mathematical Modeling]) and was due mainly to the faster exchange of PC in neuroblast interphase and the very slow dissociation of PC in SOP metaphase.

The assumption made above-that all parameters change at the same moment upon NEBD-is sufficient to explain dissociation in neuroblasts but proved to be invalid for SOPs (Fig 5C). We thus evaluated whether a changed order of events could accelerate mitotic dissociation. The moment of NEBD is well defined in our experiments (visible as dispersal of GFP fusion proteins in Figs. 2, 3; Supplemental Movies S1-S4). Simulations, including diffusion (data not shown), predicted that protein dilution occurs within seconds after NEBD, thus the assumption that protein dilution occurs effectively instantaneously upon NEBD is valid. However, the condensation and modification of chromatin begin at prophase, well before NEBD (Nowak and Corces 2004). To simulate this gradual change in the nature of the chromatin template, we introduced a gradual change in the association rate $k_{\text {on }}$, starting at prophase (Fig. 5C, red line). In both neuroblasts and SOPs, this resulted in rapid dissociation of PC before NEBD and full dissociation to the measured levels at metaphase. In summary, this analysis predicts a slower dissociation kinetic for PC in SOPs than in neuroblasts, which can be accelerated by modeling a reduction in the association rate during prophase.

\section{H3S28 phosphorylation during prophase may accelerate PC mitotic dissociation}

We next asked what molecular mechanisms could account for a change in the association rate of PC to chromatin during prophase. We reasoned that phosphorylation of Ser 28 on histone H3 (H3S28p) may be a good candidate. H3S28 phosphorylation is present during interphase and increases during mitosis (Goto et al. 1999;
Giet and Glover 2001). PcG proteins have a reduced ability to bind to the $\mathrm{H} 3$ tail trimethylated at Lys 27 (H3K27me3) if the adjacent H3S28 site is phosphorylated (Gehani et al. 2010; Lau and Cheung 2011). We reasoned that an accumulation of $\mathrm{H} 3 \mathrm{~K} 27 \mathrm{me} 3 / \mathrm{S} 28 \mathrm{p}$ during prophase could effectively reduce the association rate of PC to chromatin by reducing the number of sites available for binding in a manner analogous to that reported for heterochromatin protein 1 (HP1) (Fischle et al. 2005; Hirota et al. 2005). To determine whether H3K27me3/ S28p accumulates during prophase in neuroblasts and SOPs, we generated an antibody that specifically recognizes the double modification (see the Materials and Methods; Supplemental Fig. S5). Figure 6 shows that robust accumulation of $\mathrm{H} 3 \mathrm{~K} 27 \mathrm{me} 3 / \mathrm{S} 28 \mathrm{P}$ was indeed detectable in prophase in both neuroblasts and SOPs before the onset of NEBD. These results are consistent with a role of this double modification in ejecting PC from chromatin during prophase.

To further examine a functional role for the H3K27me3S28p double mark in regulating PC binding, we performed RNAi-mediated knockdown of the $J I L-1$ kinase in neuroblasts. $I I L-1$ is a homolog of the MSK kinase, which phosphorylates both H3S10 and H3S28 in interphase (Jin et al. 1999; Gehani et al. 2010; Lau and Cheung 2011). JIL-1 knockdown had no effect on the expression levels of PC::GFP (data not shown). Remarkably, knockdown of $I I L-1$ led to an increase in the residence time of $\mathrm{PC}:$ GFP on interphase chromatin (Fig. 6S), consistent with a role for H3S28p in ejecting PC from chromatin. We conclude that this modification may contribute to the active reduction in PC binding that we predicted by mathematical modeling to be required before $\mathrm{NEBD}$, thus accelerating mitotic dissociation of PC.

\section{Discussion}

We used a combination of quantitative live imaging and mathematical modeling to investigate changes in the dynamic behavior of PcG proteins upon mitosis and cell fate transitions in living Drosophila, giving quantitative insight into the properties of the PcG system.

For the PH::GFP fusion protein, the use of limited tissue-specific expression strategies was necessary to avoid cell death associated with $\mathrm{PH}$ overexpression. This, in turn, precluded the quantification of endogenous $\mathrm{PH}$ molecule numbers, since protocols for the isolation of GFP-marked SOPs and neuroblasts are not currently available. A goal of future studies will be to isolate the $\mathrm{PH}:$ :GFP-expressing cell types of interest in order to enable relative quantification of $\mathrm{PH}: \mathrm{GFP}$ and endogenous PH. For the PC::GFP fusion protein, the transgene was expressed under the endogenous $P c$ promoter, enabling quantification of relative amounts of transgenic and endogenous protein from whole tissues. It is important to consider to what extent the partial rescue of $P c$ mutants by the PC::GFP transgene will affect the quantitative conclusions we draw here. By quantitative comparison with $\mathrm{PH}:$ GFP behavior, it has been proposed that 

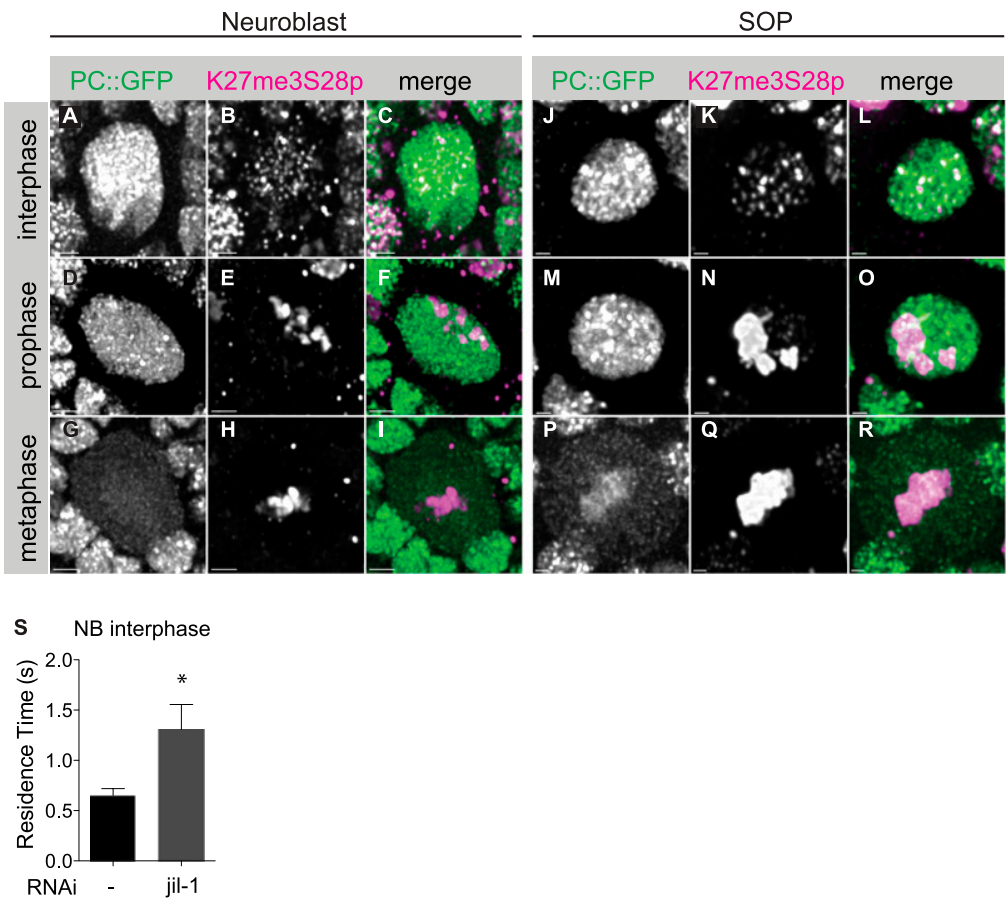

Figure 6. The H3K27me3/S28p double mark accumulates during prophase in neuroblasts and SOPs. Neuroblasts $(A-I)$ and SOPs $(J-R)$ of larvae and pupae expressing PC::GFP were fixed and stained with $\alpha \mathrm{H} 3 \mathrm{~K} 27 \mathrm{me} 3 / \mathrm{S} 28 \mathrm{p}$ (see the Materials and Methods). $(A, D, G, I, M, P)$ The GFP signal reveals PC::GFP distribution and identifies cells that have not yet undergone NEBD, visible as distinct nuclear and cytoplasmic regions in $A, D, J$, and $M .(G, P)$ After NEBD, the PC::GFP signal is dispersed throughout the cell volume. Detection of H3K27me3/S28p reveals that the double mark is present in interphase $(B, K)$ and accumulates during prophase, prior to $\operatorname{NEBD}(E, N)$. $(H, \mathrm{Q})$ Maximum levels of double mark are detected at metaphase. Bars: $A-I, 2 \mu \mathrm{m} ; J-R, 1 \mu \mathrm{m}$. (S) $I I L-1$ knockdown modulates PC::GFP kinetics in neuroblasts. The residence time of PC::GFP in neuroblast interphase upon no RNAi (-) and knockdown of $J I L-1$. Transient expression of a UAS -JIL-1 RNAi construct (Dietzl et al. 2007) (obtained from the Vienna Drosophila RNAi Center [VDRC], http:// stockcenter.vdrc.at/control/main) was achieved using an inscuteable GAL4 driver line for expression in neuroblasts (Kraut and Campos-Ortega 1996) in combination with GAL80ts (see the Materials and Methods) to achieve transient expression of the RNAi construct in third instar larvae. Data show the mean and SEM of at least three experiments. $\left.{ }^{*}\right) P$-value $<0.05$ (two-tailed $t$-test). Residence times were extracted from FRAP experiments as shown in Figure 4. Fitting was performed with fixed $D f[D f(1)]$ (Supplemental Table S1).

the PC::GFP fusion is less favored by fourfold to fivefold in the PRC1 complex than the endogenous protein (Ficz et al. 2005). Ficz et al. (2005) concluded that the population of PRC1 is marked with PC::GFP, but the bound fraction of PC::GFP may be an underestimation of the bound fraction of endogenous PC. This effect may lead to the lower bound fraction that we measure for PC::GFP in comparison with PH::GFP. It also follows from this that second-order kinetic processes (on rates) will be prone to inaccuracies, but first-order processes (off rates and therefore residence times) will be unaffected. We note that the accurate determination of the true on rate $\left(k_{\text {on }}\right)$ from the pseudo-first-order association rate $\left(k^{*}{ }_{\text {on }}\right)$, extracted from FRAP experiments such as these, is also limited by the unknown quantity of free binding sites (McNally 2008); thus, at best, one can extract relative $k_{o n}$ values that allow comparisons between different cell types. This in itself allows meaningful comparisons. In summary, we conclude that the PC::GFP fusion protein is a useful reporter of specific aspects of endogenous protein behavior: It enables the accurate determination of residence times, absolute protein quantities (which do not rely on protein activity), and relative differences between on rates in different cell types and at different cell cycle stages.

Comparison of PC::GFP and PH::GFP revealed twofold to ninefold longer residence times for PH::GFP than PC::GFP at interphase in all cell types. This result suggests that PC and PH do not solely operate as part of the PRC1 complex, consistent with previous studies (Buchenau et al. 1998). The longer residence times observed for PH::GFP may reflect multimerization of $\mathrm{PH}$ via the SAM domain, which has been shown to be required for PH-mediated gene silencing (Robinson et al. 2012). The cell type-specific differences that we observe in the kinetic behavior of PH::GFP raise the intriguing possibility that some of these may be due to regulation of sterile $\alpha$ motif (SAM) domain polymerization and thus $\mathrm{PH}$ silencing properties.

The estimation of the number of endogenous PC molecules bound to chromatin in interphase ( 25007500 depending on cell type) (Supplemental Table S1) allows comparison with numbers of PcG target genes estimated from profiling studies (between 400 and 2000) (Martinez et al. 2009; Enderle et al. 2011). We note that the interphase residence times for both proteins measured in this study $(0.5-10 \mathrm{sec})$ (Supplemental Table S1) are shorter than those previously reported for the same fusion proteins in other tissues (2-6 min) (Ficz et al. 2005). These differences may arise from the different cell types examined or from the different FRAP analysis models used. Indeed, the residence times measured here are consistent with those measured for several transcription factors using similar FRAP models (Mueller et al. 2008). These findings suggest that in interphase, several PC molecules are bound to a given target gene and exchange within a matter of seconds on a time scale similar to transcription factor-binding events. The fact that we measured shorter residence times in neuroblasts than in SOPs suggests that the mode of PcG binding, and thus the extent of silencing, may be differently regulated in stem cells and differentiated cells. 
The analysis of different cell lineages and of interphaseto-mitotic transitions led to two key findings. First, we document a progressive reduction in mobility of both PC::GFP and PH::GFP upon lineage commitment both between cell types and within a single lineage, consistent with and extending previous studies showing reduced mobility of these proteins at later developmental stages (Ficz et al. 2005) and a general loss of chromatin plasticity upon embryonic stem (ES) cell differentiation (Meshorer et al. 2006). Interestingly, a recent study of TFIIH binding in developing mammalian tissues, performed in living mice, revealed a differentiation-driven reduction in TFIIH mobility, revealing long-lasting but reversible immobilization in post-mitotic cells (Giglia-Mari et al. 2009). It will be of great interest in the future to examine $\mathrm{PC}, \mathrm{PH}$, and other PcG and TrxG (Trithorax group) proteins in other cell lineages to determine the extent to which residence times are modulatable upon changes in cell identity. In particular, it will be interesting to examine the kinetics of the DNA-binding proteins that recruit the PcG and TrxG proteins to their sites of action.

Second, we identify a fraction of PcG molecules that remain strongly bound to mitotic chromatin in both neuroblasts and SOPs. The long residence times (up to several minutes) of this bound fraction raise the important question of whether these molecules are carriers of mitotic memory. Thus, how the mitotic chromatinbinding properties of the PcG are differently regulated in SOPs and neuroblasts will be a key question for future studies. Does a strongly bound subpopulation exist in interphase? In the mathematical model for PC dissociation (Fig. 5), all PC molecules are treated as belonging to a single population whose properties change upon entry into mitosis. We note that a model in which a subpopulation with long residence time exists during interphase would also be compatible with the observed data, but such a subpopulation was not discernible from the FRAP recovery data.

The determination of molecule numbers, concentrations, and kinetic constants gives insight into the absolute quantities and mobilities of free and bound PC molecules in specific cell types in the endogenous situation, thus providing in vivo quantitation of an epigenetic system. These in vivo measurements will be essential for interpretation of models based on in vitro findings. Furthermore, this analysis enabled us to use quantitative mathematical modeling to examine the predicted behavior of the system over time during an entire cell cycle. The most important insight provided by the model is the requirement for accelerated PC displacement in SOPs and the prediction that this may be provided by a reduction in association rate during prophase. We demonstrate that H3S28 phosphorylation is a good candidate mechanism for PC displacement during prophase and metaphase, in addition to its documented role in PcG displacement during interphase (Gehani et al. 2010; Lau and Cheung 2011). The increased residence time that we observed for PC::GFP upon RNAi-mediated knockdown of $J I L-1$ is consistent with a role of $\mathrm{H} 3 \mathrm{~S} 28 \mathrm{P}$ in ejecting PC from H3K27me3 sites on chromatin. Our observation of accu- mulation of this double mark in prophase and metaphase is consistent with observations of mitotic accumulation of H3K9me3/S10p (Fischle et al. 2005; Hirota et al. 2005) but is in contrast to the study of Gehani et al. (2010), who report only slight changes in levels of H3K27me3/S28p from interphase to metaphase in human fibroblasts. This discrepancy strongly suggests that the extent of mitotic S28 phosphorylation on K27-methylated H3 tails is cell type-specific, consistent with a potential role for this mark in distinguishing the mitotic behavior of PC in SOPs and neuroblasts.

Since H3K27me3/S28p is associated with ejection of PC from chromatin, and the double mark is highly enriched on mitotic chromatin, additional mechanisms must contribute to the increased residence times of the small bound fraction of PC::GFP that we observed in mitosis. These may include post-translational modifications of PC and PH proteins themselves (Niessen et al. 2009), a switch of binding platform (e.g., from histone tails to DNA or RNA), and modification of recruiting or competing molecules (Fig. 7). Whether these proposed mechanisms contribute to mitotic PcG displacement and retention and whether they are regulated differently in different lineages will be key questions for future studies.

In summary, we demonstrate here that the properties of the PcG proteins are not only different in different lineages, but also profoundly altered at mitosis. We propose that this regulation of PcG properties may be essential to both the stability of determined cell identities and the flexibility of the stem cell state.

The combination of absolute quantification with analysis in living animals that we used in this study offers

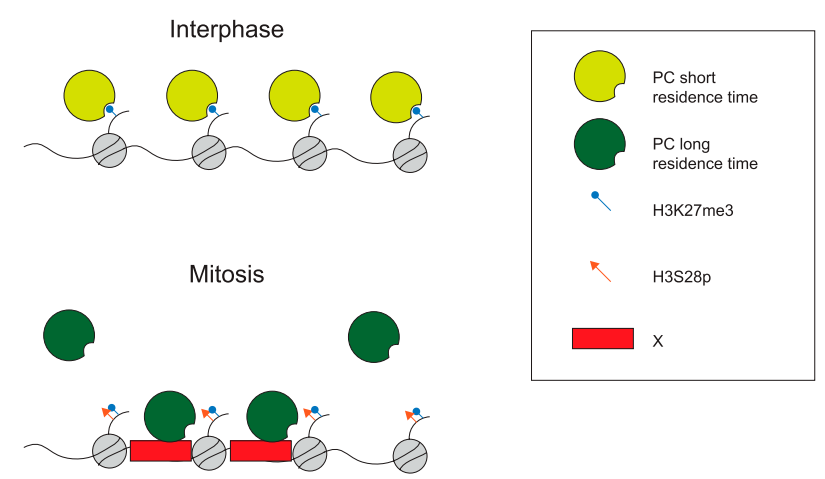

Figure 7. Model for interaction of PC with chromatin during mitosis. During interphase, PC binds chromatin through the interaction of its chromodomain with histone $\mathrm{H} 3$ trimethylated at Lys 27 (H3K27me3). As cells enter mitosis, phosphorylation of H3S28 reduces the available binding sites for PC, which, in parallel with the protein dilution at NEBD, leads to the dispersion of PC from mitotic chromatin. Additional cell type-specific processes $(\mathrm{X})$ lead to a change in the overall binding properties of PC at metaphase, so that the remaining binding PC molecules are able to bind longer to chromatin. Such mechanisms may include post-translational modifications of PcG proteins themselves, a switch of binding platform (e.g., from histone tails to DNA or RNA), and modification of recruiting or competing molecules. 
three key advances to the study of epigenetic regulation: First, we examine single, defined, genetically marked cell lineages as they go through mitosis and differentiation or self-renewal. Only in a living animal can we observe a defined mitotic event and its differentiated or selfrenewed daughter cells. Second, only by quantifying absolute numbers of chromatin-bound endogenous molecules in real volumes can we begin to understand the biological meaning of observed differences in terms of cellular concentrations and protein abundance. Third, these quantitative measurements enable not only the comparison of dynamic transitions in different cell types, but also meaningful mathematical models, identifying which parameters of the system can best explain the observed changes in the plasticity of PcG-chromatin binding upon mitosis and differentiation in stem cells and in more determined lineages. In summary, the combined use of live imaging and mathematical modeling in genetically tractable, dynamically changing in vivo experiments provides quantitative insight into how a system whose components are in constant flux can ensure both stability and flexibility.

\section{Materials and methods}

Fly strains, husbandry, and rescue experiments

The strains used in this study are shown in Table 1. Both PC and PH were used as EGFP fusion proteins (designated PC::GFP and $\mathrm{PH}:$ :GFP).

Flies were maintained in standard medium at $18^{\circ} \mathrm{C}$. Rescue experiments were performed at $25^{\circ} \mathrm{C}$, according to the scheme shown in Figure 1. For SOP lineage imaging of PH::GFP, PC::GFP, and GFPnls, crosses were made between the neuralized (neur)GAL4 driver and $P H:: G F P, P C:: G F P$, and GFPnls flies to obtain the following respective progenies: $W^{1118} ; P\left\{\right.$ Tub-GAL80 $\left.0^{t s}, w^{+}\right\}$ $P\left\{U A S, p P c-P H:: G F P, w^{+}\right\} ; P\left\{\right.$ neur-GAL4, $\left.w^{+}\right\}, P\{U A S-H 2 A:: m R F P$, $\left.w^{+}\right\} /+. w^{1118} ; P\left\{\right.$ Tub-GAL80 $\left.0^{t s}, w^{+}\right\} /+; P\left\{\right.$ neur-GAL4, $\left.w^{+}\right\}, P\{U A S-$ H2A::mRFP, $\left.w^{+}\right\} / P\left\{p P c-P C:: G F P, w^{+}\right\} . w^{1118} ; P\left\{T u b-G A L 80^{t s}, w^{+}\right\}$ P $\left\{U A S\right.$-GFPnls, $\left.w^{+}\right\} ;$P $\left\{\right.$neur-GAL4, $\left.w^{+}\right\}, P\left\{U A S-H 2 A:: m R F P, w^{+}\right\} /+$. These flies were switched to $25^{\circ} \mathrm{C} 15 \mathrm{~h}$ before SOP imaging and FRAP to allow for GAL4-driven expression. For neuroblast lineage imaging of $\mathrm{PH}: \mathrm{GFP}, P H:: G F P, H 2 A:: R F P$ third instar larvae were collected and imaged without further crossing. For neuroblast lineage imaging of PC::GFP, a cross between $H 2 A:: R F P$ and $P C:: G F P, H 2 A:: R F P$ flies was conducted to obtain the following genotype: $w^{1118} ;+; P\left\{p P c-P C:: G F P, w^{+}\right\}, P\{U b i-$ $\left.H 2 A:: m R F P, w^{+}\right\} / P\left\{U b i-H 2 A:: m R F P, w^{+}\right\}$For neuroblast imaging of GFPnls, a cross between GFPnls and H2A::RFP flies was conducted to obtain the following genotype: $w^{1118}$; $P\{U A S$ GFPnls, $\left.w^{+}\right\} /+$; $P\left\{U b i-H 2 A:: m R F P, w^{+}\right\} /+$. For RNAi knockdown of $J I L-1$, Insc-driver female flies were crossed to $I I L-1$ RNAi males and raised at $18^{\circ} \mathrm{C}$. Upon appearance of third instar larvae, the progeny was transferred for $24-48 \mathrm{~h}$ to $29^{\circ} \mathrm{C}$. H2A::RFP-expressing neuroblasts were then used for subsequent experiments.

\section{Quantitative Western blotting}

Fly extracts and Western blots were performed as described in Ficz et al. (2005) with minimal changes. Proteins were separated in NuPAGE $4 \%-12 \%$ Bis-Tris, and PC was probed with a primary polyclonal $\alpha$-PC antibody (d-220, Santa Cruz Biotechnology) and histone $\mathrm{H} 3$ with a primary polyclonal $\alpha-\mathrm{H} 3$ antibody (Abcam). HRP-conjugated antibodies were used for secondary detection. ECL-Plus (Amersham) detection was used to generate chemofluorescence detected and quantified using a Typhoon scanner (GE Healthcare). All values were normalized to a loading control.

\section{H3K27me3S28ph antibody production}

A rabbit polyclonal H3K27me3S28ph antibody was generated in collaboration with Eurogentec. Animals were immunized with the KLH-conjugated synthetic peptide QLATKAARK(me3) S(ph)APATGGVKKC corresponding to amino acids 19-37 of histone H3. Positive sera were purified by the manufacturer in a two-step procedure. Antibodies specific to the dual modification were captured on the AF-Amino TOYOPEARL $650 \mathrm{M}$ matrix coupled with the peptide used for immunization: QLATKAARK(me3) S(ph)APATGGVKKC. Following elution with $100 \mathrm{mM}$ glycine $(\mathrm{pH}$ $2.5)$, antibodies recognizing the peptide in the absence of modifications were removed with a matrix coupled with the unmodified peptide QLATKAARKSAPATGGVKKC. After the purification, antibody specificity was tested by dot blotting.

Table 1. Strains used in this study

\begin{tabular}{|c|c|c|}
\hline Name & Genotype & Source \\
\hline PC::GFP & $w^{1118} ;+; P\left\{p P c-P C:: G F P, w^{+}\right\}$ & R. Paro \\
\hline PH::GFP & $w^{1118} ; P\left\{U A S, p P c-P H:: G F P, w^{+}\right\} ;+$ & D. Arndt-Jovin \\
\hline GFPnls & $w^{1118} ; P\left\{U A S-G F P n l s, w^{+} ;+\right\}$ & B. Dickson \\
\hline neur driver & $w^{1118} ; P\left\{\right.$ Tub-GAL80 $\left.0^{t s}, w^{+}\right\} ; P\left\{\right.$ neur-GAL4, $\left.w^{+}\right\}, P\left\{U A S-H 2 A:: m R F P, w^{+}\right\}$ & J. Knoblich \\
\hline H2A::RFP & 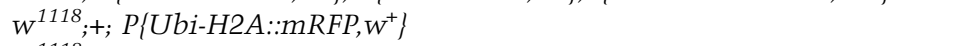 & J. Knoblich \\
\hline PC::GFP, H2A::RFP & $w^{1118} ;+; P\left\{p P c-P C:: G F P, w^{+}\right\}, P\left\{U b i-H 2 A:: m R F P, w^{+}\right.$ & \\
\hline PH::GFP, H2A::RFP & $\begin{array}{l}w^{1118} ; P\left\{U A S, p P c-P H:: G F P, w^{+}\right\}, P\left\{\text { worn-GAL4, } w^{+}\right\} / C y, O \\
\quad P\left\{U b i-H 2 A:: m R F P, w^{+}\right\}\end{array}$ & \\
\hline $\begin{array}{l}\text { GFPnls, H2A::RFP } \\
\text { Pc }\end{array}$ & $\begin{array}{l}w^{1118} ; P\left\{U A S-G F P n l s, w^{+}\right\}, P\left\{\text { worn-GAL4, } w^{+}\right\} / C y, O ; P\left\{U b i-H 2 A:: m R F P, w^{+}\right\} \\
w^{1118} ;+; P c^{X L 5} / T M 3, S e r\end{array}$ & \\
\hline $\mathrm{Pc}^{3}$ & $\operatorname{In}(3 R) P\left(P c^{3}\right), P c^{3 /} T M 1$ & $\begin{array}{l}\text { Drosophila } \\
\text { Genomics Resource } \\
\text { Center no. } 106475\end{array}$ \\
\hline PC::GFP (II) & $w^{1118} ; P\left\{p P c-P C:: G F P, w^{+}\right\} ;+$ & R. Paro \\
\hline Insc driver & $w^{1118} ; P\left\{\right.$ neur-GAL4, $\left.w^{+}\right\} / C y, O ; P\left\{\right.$ Tub-GAL80 $\left.0^{t s}, w^{+}\right\}$ & J. Knoblich \\
\hline JIL-1 RNAi & $\begin{array}{l}\text { w1118; }\{U A S-J I L-1 \text { RNAi }(V D R C \# 107001 K K)\}, w^{+} ; P\{p P c-P C:: G F P, w+\}, \\
\quad P\{U b i-H 2 A:: m R F P, w+\}\end{array}$ & $\begin{array}{l}\text { VDRC no. } \\
107001 \mathrm{KK}\end{array}$ \\
\hline
\end{tabular}




\section{Dot blot}

Synthetic peptides corresponding to the $\mathrm{N}$-terminal sequence of histone H3 (amino acids 19-37), with different S28 phosphorylation and K27 methylation status, were spotted on a PVDF membrane and probed with the a-H3K27me3S28ph antibody (dilution 1:20,000). For detection, a secondary anti-rabbit horseradish peroxidase-conjugated antibody and the Enhanced Chemiluminescence (ECL) detection system were used. To ensure equal peptide loading, a duplicate membrane was stained with Ponceau S (Supplemental Fig. S5).

\section{Immunocytochemistry in larval brains and pupal nota}

Immunocytochemistry experiments in larval brains were performed as described (Betschinger et al. 2006). Immunocytochemistry experiments in pupal nota were performed by dissecting the pupae in $5 \%$ paraformaldehyde in phosphate-buffered saline (PBS). The total time of fixation was $20 \mathrm{~min}$, and the subsequent procedure was identical to that described for larval brains. The primary antibodies used were rabbit $\alpha$-Miranda (1:250) (Betschinger et al. 2006) and rabbit $\alpha-\mathrm{H} 3 \mathrm{~K} 27 \mathrm{me} 3 \mathrm{~S} 28 \mathrm{ph}$ (1:2000) (this study). The secondary antibody was AlexaFluor 647 goat $\alpha$-rabbit (1:500) (Invitrogen A-21245). For the images shown in Figure 6, GFP was imaged directly without staining.

\section{Quantification of GFP fusion proteins using GFP-VLPS}

For quantification of EGFP-fused proteins, EGFP-VLPs $(100 \mathrm{ng} / \mu \mathrm{L})$ were spread on a \#1.5 coverslip and imaged under the same settings as the GFP fusion protein of interest. Images of GFPVLPs were deconvolved with the CMLE method of the software Huygens Pro (SVI), and a total intensity of at least 80 particles was determined using Imaris (Bitplane). After binning of intensities, the mode of the distribution was selected as the intensity of 120 GFP molecules. Comparison of GFP fusion intensities calculated with Imaris (Bitplane) with this value allowed the estimation of GFP fusion protein quantities.

\section{Quantification of GFP fusion proteins using ELISA}

ELISA was performed using a GFP ELISA kit (Cell Biolabs), according to the manufacturer's instructions. Total protein was extracted from 20 hand-collected, dechorionated, 2:15- to 2:45-hold embryos by sonification in $100 \mu \mathrm{L}$ of DLB buffer $(20 \mathrm{mM}$ HEPES-KOH at $\mathrm{pH} 7.5,100 \mathrm{mM} \mathrm{KCl}, 2 \mathrm{mM}$ EDTA, 0.5\% Triton $\mathrm{X}-100,5 \mathrm{mM}$ DTT, $1 \mathrm{mM} \mathrm{MgAc}$, Roche Complete protease inhibitors). Extracts were cleared by centrifugation and diluted 20 -fold using assay diluent (GFP ELISA kit). Samples were measured in duplicates. The number of GFP molecules per nucleus was calculated based on the observation that each embryo contains 6000 nuclei (Foe and Alberts 1983) and that $100 \mu \mathrm{L}$ of diluted extract corresponds to the protein from one embryo. The number of PC::EGFP molecules per nucleus was calculated using the following equation: molecules $=($ mass of PC::GFP $[\mathrm{g}] / \mathrm{MW}$ GFP) $\times\left(6.022 \times 10^{-23}\right) / 6000$ nuclei.

\section{Preparation of specimens for microscopy and imaging}

For SOP imaging, pupae were prepared as described in Bellaiche et al. (2001). Imaging was performed at room temperature on a Zeiss LSM710 with a $63 \times 1.4$ NA oil immersion objective using an Argon laser at $488 \mathrm{~nm}$ for GFP imaging (collection between 485 and $563 \mathrm{~nm}$ ) and a DPSS 561-10 laser at $561 \mathrm{~nm}$ for mRFP imaging (collection between 563 and $728 \mathrm{~nm}$ ) and a pinhole equivalent to 2.45 Airy units. The voxel size was $51 \times 51 \times 150 \mathrm{~nm}$.
For neuroblast imaging, larval brains were dissected in a Lab-Tek II Chamber \#1.5 German Coverglass (Nalgene Nunc International) containing $200 \mu \mathrm{L}$ of PBS. Imaging was performed at room temperature on a Zeiss LSM710 with a $63 \times 1.2$ NA water immersion objective using an Argon laser at $488 \mathrm{~nm}$ for GFP imaging (collection between 485 and $563 \mathrm{~nm}$ ) and a DPSS 561-10 laser at $561 \mathrm{~nm}$ for mRFP imaging (collection between 563 and $728 \mathrm{~nm}$ ) and a pinhole equivalent to 2.07 Airy units. The voxel size was $51 \times 51 \times 150 \mathrm{~nm}$.

\section{Image processing}

All images acquired were deconvolved using measured PSFs with the CMLE method of the software Huygens Pro (SVI). The measurement of the volumes occupied and the total fluorescence intensity of GFP and mRFP were performed with Imaris (Bitplane). Background was subtracted from intensity measurements.

\section{FRAP}

FRAP experiments were performed as described in Mueller et al. (2008) with minimal changes. FRAP was performed in a Zeiss LSM710 with the confocal pinhole set to 3 Airy units. The image size was $512 \times 62$ pixels, with a pixel size of $45 \mathrm{~nm}$. Images were acquired every $20 \mathrm{msec}$ for GFPnls and PC::GFP experiments and $50 \mathrm{msec}$ and $100 \mathrm{msec}$ for PH::GFP using 1\% AOTF of the 488 line of an Argon laser. The circle bleach region had a diameter of 40 pixels; it was centered on the image field and overlapped with expression of $\mathrm{H} 2 \mathrm{~A}:: \mathrm{RFP}$. The bleach iteration number was set to 2 and $100 \%$ AOTF of the 488 line in an Argon laser. Background was measured within a region with no cells of the image and subtracted from data. All intensity measurements were performed with MatLab (Mathworks).

\section{FRAP model fitting}

FRAP modeling of recovery curves was performed according to Mueller et al. (2008) in MatLab (Mathworks) with minimal changes, described in detail in the Supplemental Material (FRAP Data Analysis). An adaptation of the model described in Beaudouin et al. (2006) for evaluation of the effect of nonhomogeneous protein distribution on extracted parameters was implemented in Mathematica (Wolfram). Cross-validation was also performed on FRAP recovery curves measured in metaphase according to Sprague et al. (2006) using Matlab (Mathworks). The above methods are described in detail in the Supplemental Material (FRAP Data Analysis).

\section{Mathematical model of PC interaction with chromatin}

A linearized ordinary differential equation system was used to model the interaction of endogenous PC proteins with chromatin. A full description of the equations and parameters of this model are given in the Supplemental Material (Mathematical Modeling) and Supplemental Table S1. Simulations were performed using MatLab (Mathworks).

\section{Acknowledgments}

We are grateful to P. Pasierbek of the IMP/IMBA biooptics facility for advice and assistance on live imaging. We are grateful to D.A.-Jovin and G. Ficz for discussions and advice on imaging. We thank F. Müller for MatLab files and advice on FRAP, and D. Poncet and A. Charpilienne for providing VLPs. We thank J. Knoblich, R. Paro, D.A.-Jovin, and B. Dickson for fly stocks. We 
thank J. Mummery-Widmer, F. Mauri, A. Fischer, and C. Homem for advice and assistance on imaging of SOP and neuroblast lineages. We are grateful to M. Baffoe-Bonnie Owusu and C. Ehrhardt for technical assistance, and to M. Rehmsmeier and Ringrose laboratory members for stimulating discussions. This work was funded by the Austrian Academy of Sciences (http://www.oeaw.ac.at) (to L.R., J.L., and S.M.), by the EU FP6 Network of Excellence, "The Epigenome" (http://www.epigenome-noe. net) (to P.S), and the EU FP7 Network of Excellence, "Epigenesys" (http://www.epigenesys.eu// (to P.S). J.P.F. is supported by the Portuguese Foundation for Science and Technology [http:// www.fct.pt (SFRH/BD/40389/2007)]. A.S. is a fellow of the International $\mathrm{PhD}$ program "Molecular Mechanism of Cell Signaling" (http://www.phd-cellular-signaling.at), supported by the Austrian Science Fund (http://www.fwf.ac.at/en). Research in the Seiser laboratory was supported by the Austrian Science Fund (FWF P22340).

\section{References}

Beaudouin J, Mora-Bermudez F, Klee T, Daigle N, Ellenberg J. 2006. Dissecting the contribution of diffusion and interactions to the mobility of nuclear proteins. Biophys J 90: 18781894.

Bellaiche Y, Gho M, Kaltschmidt JA, Brand AH, Schweisguth F. 2001. Frizzled regulates localization of cell-fate determinants and mitotic spindle rotation during asymmetric cell division. Nat Cell Biol 3: 50-57.

Bello B, Holbro N, Reichert H. 2007. Polycomb group genes are required for neural stem cell survival in postembryonic neurogenesis of Drosophila. Development 134: 10911099.

Betschinger J, Mechtler K, Knoblich JA. 2006. Asymmetric segregation of the tumor suppressor brat regulates self-renewal in Drosophila neural stem cells. Cell 124: 1241-1253.

Buchenau P, Hodgson J, Strutt H, Arndt-Jovin DJ. 1998. The distribution of polycomb-group proteins during cell division and development in Drosophila embryos: Impact on models for silencing. J Cell Biol 141: 469-481.

Chen X, Hiller M, Sancak Y, Fuller MT. 2005. Tissue-specific TAFs counteract Polycomb to turn on terminal differentiation. Science 310: 869-872.

Dietzel S, Niemann H, Bruckner B, Maurange C, Paro R. 1999. The nuclear distribution of Polycomb during Drosophila melanogaster development shown with a GFP fusion protein. Chromosoma 108: 83-94.

Dietzl G, Chen D, Schnorrer F, Su KC, Barinova Y, Fellner M, Gasser B, Kinsey K, Oppel S, Scheiblauer S, et al. 2007. A genome-wide transgenic RNAi library for conditional gene inactivation in Drosophila. Nature 448: 151-156.

Doe CQ. 2008. Neural stem cells: Balancing self-renewal with differentiation. Development 135: 1575-1587.

Dundr M, McNally JG, Cohen J, Misteli T. 2002. Quantitation of GFP-fusion proteins in single living cells. I Struct Biol 140: 92-99.

Enderle D, Beisel C, Stadler MB, Gerstung M, Athri P, Paro R. 2011. Polycomb preferentially targets stalled promoters of coding and noncoding transcripts. Genome Res 21: 216-226.

Fanti L, Perrini B, Piacentini L, Berloco M, Marchetti E, Palumbo G, Pimpinelli S. 2008. The trithorax group and Pc group proteins are differentially involved in heterochromatin formation in Drosophila. Chromosoma 117: 25-39.

Ficz G. 2005. "Protein dynamics in the nucleus: Implications for gene expression." $\mathrm{PhD}$ thesis. Georg-August-Universität, Göttingen, Germany.
Ficz G, Heintzmann R, Arndt-Jovin DJ. 2005. Polycomb group protein complexes exchange rapidly in living Drosophila. Development 132: 3963-3976.

Fischle W, Tseng BS, Dormann HL, Ueberheide BM, Garcia BA, Shabanowitz J, Hunt DF, Funabiki H, Allis CD. 2005. Regulation of HP1-chromatin binding by histone H3 methylation and phosphorylation. Nature 438: 1116-1122.

Foe VE, Alberts BM. 1983. Studies of nuclear and cytoplasmic behaviour during the five mitotic cycles that precede gastrulation in Drosophila embryogenesis. I Cell Sci 61: 31-70.

Gehani SS, Agrawal-Singh S, Dietrich N, Christophersen NS, Helin K, Hansen K. 2010. Polycomb group protein displacement and gene activation through MSK-dependent H3K27me3S28 phosphorylation. Mol Cell 39: 886-900.

Giet R, Glover DM. 2001. Drosophila aurora B kinase is required for histone $\mathrm{H} 3$ phosphorylation and condensin recruitment during chromosome condensation and to organize the central spindle during cytokinesis. J Cell Biol 152: 669-682.

Giglia-Mari G, Theil AF, Mari PO, Mourgues S, Nonnekens J, Andrieux LO, de Wit J, Miquel C, Wijgers N, Maas A, et al. 2009. Differentiation driven changes in the dynamic organization of Basal transcription initiation. PLOS Biol 7: e1000220. doi: 10.1371/journal.pbio.1000220.

Goto H, Tomono Y, Ajiro K, Kosako H, Fujita M, Sakurai M, Okawa K, Iwamatsu A, Okigaki T, Takahashi T, et al. 1999. Identification of a novel phosphorylation site on histone $\mathrm{H} 3$ coupled with mitotic chromosome condensation. I Biol Chem 274: 25543-25549.

Gottesfeld JM, Forbes DJ. 1997. Mitotic repression of the transcriptional machinery. Trends Biochem Sci 22: 197-202.

Hirota T, Lipp JJ, Toh BH, Peters JM. 2005. Histone H3 serine 10 phosphorylation by Aurora B causes HP1 dissociation from heterochromatin. Nature 438: 1176-1180.

Jin Y, Wang Y, Walker DL, Conley C, Johansen J, Johansen KM. 1999. IIL-1: A novel chromosomal tandem kinase implicated in transcriptional regulation in Drosophila. Mol Cell 4: 129-135.

Kraut R, Campos-Ortega JA. 1996. inscuteable, a neural precursor gene of Drosophila, encodes a candidate for a cytoskeleton adaptor protein. Dev Biol 174: 65-81.

Kwong C, Adryan B, Bell I, Meadows L, Russell S, Manak JR, White R. 2008. Stability and dynamics of Polycomb target sites in Drosophila development. PLoS Genet 4: e1000178. doi: 10.1371/journal.pgen.1000178.

Lau PN, Cheung P. 2011. Histone code pathway involving H3 S28 phosphorylation and K27 acetylation activates transcription and antagonizes polycomb silencing. Proc Natl Acad Sci 108: 2801-2806.

Lee CY, Andersen RO, Cabernard C, Manning L, Tran KD, Lanskey MJ, Bashirullah A, Doe CQ. 2006. Drosophila Aurora-A kinase inhibits neuroblast self-renewal by regulating aPKC/Numb cortical polarity and spindle orientation. Genes Dev 20: 3464-3474.

Martinez AM, Schuettengruber B, Sakr S, Janic A, Gonzalez C, Cavalli G. 2009. Polyhomeotic has a tumor suppressor activity mediated by repression of Notch signaling. Nat Genet 41: 1076-1082.

Martinez-Balbas MA, Dey A, Rabindran SK, Ozato K, Wu C. 1995. Displacement of sequence-specific transcription factors from mitotic chromatin. Cell 83: 29-38.

Mayer B, Emery G, Berdnik D, Wirtz-Peitz F, Knoblich JA. 2005. Quantitative analysis of protein dynamics during asymmetric cell division. Curr Biol 15: 1847-1854.

McNally JG. 2008. Quantitative FRAP in analysis of molecular binding dynamics in vivo. Methods Cell Biol 85: 329-351. 
Meshorer E, Yellajoshula D, George E, Scambler PJ, Brown DT, Misteli T. 2006. Hyperdynamic plasticity of chromatin proteins in pluripotent embryonic stem cells. Dev Cell 10: $105-116$

Mohn F, Weber M, Rebhan M, Roloff TC, Richter J, Stadler MB, Bibel M, Schubeler D. 2008. Lineage-specific polycomb targets and de novo DNA methylation define restriction and potential of neuronal progenitors. Mol Cell 30: 755-766.

Mueller F, Wach P, McNally JG. 2008. Evidence for a common mode of transcription factor interaction with chromatin as revealed by improved quantitative fluorescence recovery after photobleaching. Biophys J 94: 3323-3339.

Mueller F, Mazza D, Stasevich TJ, McNally JG. 2010. FRAP and kinetic modeling in the analysis of nuclear protein dynamics: What do we really know? Curr Opin Cell Biol 22: 403411.

Mummery-Widmer JL, Yamazaki $M$, Stoeger T, Novatchkova M, Bhalerao S, Chen D, Dietzl G, Dickson BJ, Knoblich JA. 2009. Genome-wide analysis of Notch signalling in Drosophila by transgenic RNAi. Nature 458: 987-992.

Neumuller RA, Knoblich JA. 2009. Dividing cellular asymmetry: Asymmetric cell division and its implications for stem cells and cancer. Genes Dev 23: 2675-2699.

Neumuller RA, Richter C, Fischer A, Novatchkova M, Neumuller KG, Knoblich JA. 2011. Genome-wide analysis of self-renewal in Drosophila neural stem cells by transgenic RNAi. Cell Stem Cell 8: 580-593.

Niessen HE, Demmers JA, Voncken JW. 2009. Talking to chromatin: Post-translational modulation of Polycomb group function. Epigenetics Chromatin 2: 10. doi: 10.1186/17568935-2-10.

Nowak SJ, Corces VG. 2004. Phosphorylation of histone H3: A balancing act between chromosome condensation and transcriptional activation. Trends Genet 20: 214-220.

Prescott DM, Bender MA. 1962. Synthesis of RNA and protein during mitosis in mammalian tissue culture cells. Exp Cell Res 26: 260-268.

Rabut G, Doye V, Ellenberg J. 2004. Mapping the dynamic organization of the nuclear pore complex inside single living cells. Nat Cell Biol 6: 1114-1121.

Ren X, Vincenz C, Kerppola TK. 2008. Changes in the distributions and dynamics of polycomb repressive complexes during embryonic stem cell differentiation. Mol Cell Biol 28: 2884-2895.

Robinson AK, Leal BZ, Chadwell LV, Wang R, Ilangovan U, Kaur Y, Junco SE, Schirf V, Osmulski PA, Gaczynska M, et al. 2012. The growth-suppressive function of the Polycomb group protein Polyhomeotic is mediated by polymerization of its sterile $\alpha$ motif (SAM) domain. I Biol Chem doi: 10.1074/jbc.M111.336115.

Sawarkar R, Paro R. 2010. Interpretation of developmental signaling at chromatin: The Polycomb perspective. Dev Cell 19: $651-661$.

Schuettengruber B, Cavalli G. 2009. Recruitment of Polycomb group complexes and their role in the dynamic regulation of cell fate choice. Development 136: 3531-3542.

Sharp EJ, Martin EC, Adler PN. 1994. Directed overexpression of Suppressor 2 of zeste and Posterior Sex Combs results in bristle abnormalities in Drosophila melanogaster. Dev Biol 161: 379-392.

Sprague BL, Muller F, Pego RL, Bungay PM, Stavreva DA, McNally JG. 2006. Analysis of binding at a single spatially localized cluster of binding sites by fluorescence recovery after photobleaching. Biophys J 91: 1169-1191.

Vincenz C, Kerppola TK. 2008. Different polycomb group CBX family proteins associate with distinct regions of chromatin using nonhomologous protein sequences. Proc Natl Acad Sci 105: 16572-16577.

Wheeler SR, Kearney JB, Guardiola AR, Crews ST. 2006. Singlecell mapping of neural and glial gene expression in the developing Drosophila CNS midline cells. Dev Biol 294: 509-524. 


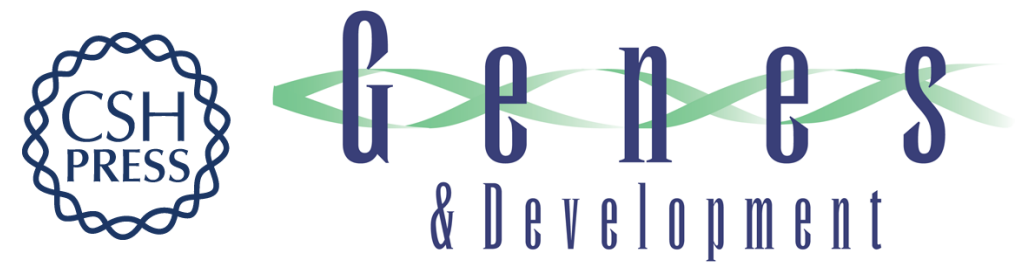

\section{In vivo Polycomb kinetics and mitotic chromatin binding distinguish stem cells from differentiated cells}

João Pedro Fonseca, Philipp A. Steffen, Stefan Müller, et al.

Genes Dev. 2012, 26:

Access the most recent version at doi:10.1101/gad.184648.111

\section{Supplemental http://genesdev.cshlp.org/content/suppl/2012/04/13/26.8.857.DC1 Material}

References This article cites 48 articles, 15 of which can be accessed free at: http://genesdev.cshlp.org/content/26/8/857.full.html\#ref-list-1

License Freely available online through the Genes \& Development Open Access option.
Email Alerting Service

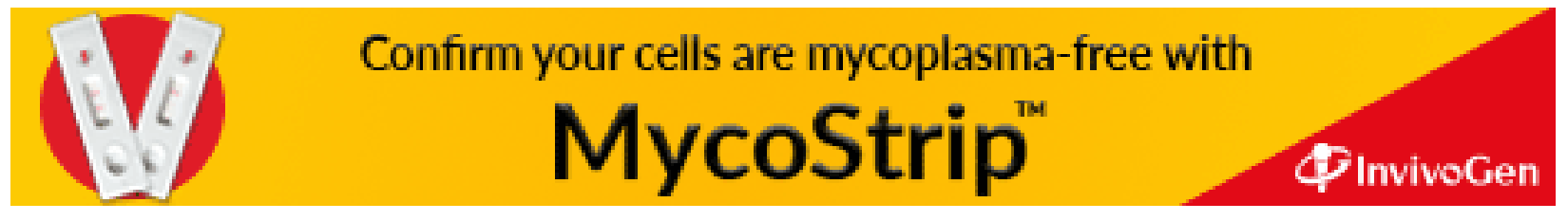

